\title{
Erratum: Unified description of efficiency correction and error estimation for moments of conserved quantities in heavy-ion collisions [Phys. Rev. C 91, 034907 (2015)]
}

\author{
Xiaofeng Luo (罗晓峰) \\ (Received 6 November 2016; published 21 November 2016)
}

DOI: 10.1103/PhysRevC.94.059901

There is a typographical error in the efficiency correction formula for the fourth order cumulant $\left(C_{4}\right)$ in Eqs. (17) in the original paper. The correct one should read

$$
\begin{aligned}
C_{4}^{X-Y}= & \frac{C_{4}^{x-y}-2(\varepsilon-1) C_{3}^{x+y}+8(\varepsilon-1)\left(C_{3}^{x}+C_{3}^{y}\right)+(5-\varepsilon)(\varepsilon-1) C_{2}^{x+y}}{\varepsilon^{4}} \\
& +\frac{8(\varepsilon-1)(\varepsilon-2)\left(C_{2}^{x}+C_{2}^{y}\right)+\left(\varepsilon^{2}-6 \varepsilon+6\right)(\varepsilon-1)(\langle x\rangle+\langle y\rangle)}{\varepsilon^{4}},
\end{aligned}
$$

where the minus sign "-_" in $C_{3}^{x-y}$ is replaced with the plus sign "+". This typographical error does not affect any of the results and conclusions in the paper. 